\title{
Capturing the P-T Evolution of Magmas in Volcanic Plumbing Systems by Machine Learning
}

\author{
Petrelli M. 1, Caricchi L. 2, Perugini D.1
}

1 University of Perugia (Italy), maurizio.petrelli@unipg.it 2 University of Geneva (Switzerland)

Clinopyroxene based thermometers and barometers are widely used tools to disclose temperature and pressure changes of magmas in volcanic plumbing systems before eruptions.

Several studies reported the development and the application of Clinopyroxene-liquid geothermobarometers in many different volcanic environments, also warning on the potential pitfall in using overly complex models [e.g., 1 and references therein]. The main drawback in the use of models with a large number of parameters is the potential overfitting of the calibration data, yielding a poor accuracy in real-world applications. On the other hand, simpler models cannot account for the complexity of natural magmatic systems, requiring different calibrations for different magma chemistries [e.g., 2, 3].

In the present study, we report on the development of Clinopyroxene and Clinopyroxene-liquid thermometers and barometers in a wide range of P-T-X conditions using Machine Learning (ML) algorithms. We define a strategy to avoid overfitting and demonstrate the robustness of the different reported methods. We compared the performance of ML algorithms with classical and established Clinopyroxene and Clinopyroxene-liquid thermometers and barometers using local and global calibrations. Finally, we applied the obtained thermometers and barometers to real study cases.

[1] K. D. Putirka, Thermometers and barometers for volcanic systems, Minerals, Inclusions and Volcanic Processes, 69. 61-120, 2008.

[2] D. A. Neave, K. D. Putirka, Am. Mineral., 2017, DOI:10.2138/am-2017-5968.

[3] M. Masotta, S. Mollo, C. Freda, M. Gaeta, G. Moore, Contrib. to Mineral. Petrol., 2013, DOI:10.1007/s00410013-0927-9. 